\title{
A Second Order Extension of the Generalized- $\alpha$ Method for Constrained Systems in Mechanics
}

\author{
Laurent O. Jay ${ }^{1}$ and Dan Negrut ${ }^{2}$ * \\ 1 Department of Mathematics, The University of Iowa, 14 MacLean Hall, Iowa \\ City, IA 52242-1419, USA, na.ljay@na-net.ornl.gov, ljay@math.uiowa.edu \\ 2 Department of Mechanical Engineering, University of Wisconsin-Madison, \\ 2035ME, 1513 University Avenue, Madison, WI 53706-1572, USA, \\ negrut@engr.wisc.edu
}

Summary. We present a new second order extension of the generalized $-\alpha$ method of Chung and Hulbert for systems in mechanics having nonconstant mass matrix, holonomic constraints, and/or nonholonomic constraints. Such systems are frequently encountered in multibody dynamics. For variable step-sizes, a new adjusting formula preserving the second order of the method is proposed.

\section{Introduction}

The generalized- $\alpha$ method of Chung and Hulbert [2] was originally developed for second order systems of differential equations in structural dynamics of the form $M y^{\prime \prime}=f\left(t, y, y^{\prime}\right)$. In mechanics $M \in \mathbb{R}^{n \times n}$ is a constant mass matrix, $y \in \mathbb{R}^{n}$ is a vector of generalized coordinates, $y^{\prime} \in \mathbb{R}^{n}$ is a vector of generalized velocities, $y^{\prime \prime} \in \mathbb{R}^{n}$ is a vector of generalized accelerations, and $f\left(t, y, y^{\prime}\right) \in \mathbb{R}^{n}$ represents forces. Introducing the new variables $z:=y^{\prime} \in \mathbb{R}^{n}$ and $a:=z^{\prime}=y^{\prime \prime} \in \mathbb{R}^{n}$, these equations are equivalent to the semi-explicit system of differential-algebraic equations (DAEs)

$$
y^{\prime}=z, \quad z^{\prime}=a, \quad 0=M a-f(t, y, z) .
$$

Assuming the mass matrix $M$ to be nonsingular, this system of DAEs is of index 1 since one can obtain explicitly $a=M^{-1} f(t, y, z)$. The generalized$\alpha$ method of Chung and Hulbert [2] for $M y^{\prime \prime}=f\left(t, y, y^{\prime}\right)$ or equivalently for (1) is a non-standard implicit one-step method. One step of this method $\left(t_{0}, y_{0}, z_{0}, a_{\alpha}\right) \mapsto\left(t_{1}=t_{0}+h, y_{1}, z_{1}, a_{1+\alpha}\right)$ with step-size $h$ can be expressed as follows

\footnotetext{
* This material is based upon work supported by the National Science Foundation under Grant No. 0654044.
} 


$$
\begin{aligned}
y_{1} & =y_{0}+h z_{0}+\frac{h^{2}}{2}\left((1-2 \beta) a_{\alpha}+2 \beta a_{1+\alpha}\right), \\
z_{1} & =z_{0}+h\left((1-\gamma) a_{\alpha}+\gamma a_{1+\alpha}\right), \\
\left(1-\alpha_{m}\right) M a_{1+\alpha}+\alpha_{m} M a_{\alpha} & =\left(1-\alpha_{f}\right) f\left(t_{1}, y_{1}, z_{1}\right)+\alpha_{f} f\left(t_{0}, y_{0}, z_{0}\right),
\end{aligned}
$$

see Section 2 for a justification of the notation $a_{\alpha}, a_{1+\alpha}$. The generalized- $\alpha$ method has free coefficients $\alpha_{m} \neq 1, \alpha_{f}, \beta, \gamma$. For specific choices of these coefficients we obtain well-known methods:

- Newmark's family: $\alpha_{m}=0, \alpha_{f}=0$;

- The trapezoidal rule: $\beta=1 / 4, \gamma=1 / 2$;

- Störmer's rule: $\beta=0, \gamma=1 / 2$;

- The Hilber-Hughes-Taylor $\alpha$ (HHT- $\alpha$ ) method [3, 4]:

$$
\alpha_{m}=0, \quad \alpha:=-\alpha_{f} \in\left[-\frac{1}{3}, 0\right], \quad \beta=\frac{(1-\alpha)^{2}}{4}, \quad \gamma=\frac{1}{2}-\alpha .
$$

The coefficients $\alpha_{m} \neq 1, \alpha_{f}, \beta, \gamma$ of the generalized- $\alpha$ method (2) are usually chosen according to

$$
\alpha_{m}=\frac{2 \rho_{\infty}-1}{1+\rho_{\infty}}, \quad \alpha_{f}=\frac{\rho_{\infty}}{1+\rho_{\infty}}, \quad \beta=\frac{(1-\alpha)^{2}}{4}, \quad \gamma=\frac{1}{2}-\alpha
$$

where $\alpha:=\alpha_{m}-\alpha_{f}$ and $\rho_{\infty} \in[0,1]$ is a parameter controlling numerical dissipation $\left(\rho_{\infty}=0\right.$ for maximal dissipation [2]).

In this paper we present extensions of the generalized- $\alpha$ method (2) for systems having

- Nonconstant mass matrix $M(t, y)$, see Section 3;

- Holonomic constraints $g(t, y)=0$, see Section 4;

- Nonholonomic constraints $k\left(t, y, y^{\prime}\right)=0$, see Section 5 .

Such systems are frequently encountered in multibody dynamics [10]. A general extension and a convergence result is given in Section 6. For variable step-sizes, a new adjusting formula preserving the second order of the method is proposed in Section 7. Some numerical experiments are given in Section 8. A short conclusion is finally given in Section 9.

\section{About the Notation $a_{\alpha}, a_{1+\alpha}$}

We use the notation $a_{\alpha}$ and $a_{1+\alpha}$ instead of $a_{0}$ and $a_{1}$ to emphasize the fact that these quantities should not be considered as approximations to the acceleration vector $a(t)$ at $t_{0}$ and $t_{1}$ respectively, but at $t_{\alpha}:=t_{0}+\alpha h$ and $t_{1+\alpha}:=t_{1}+\alpha h=t_{0}+(1+\alpha) h$ respectively where $\alpha:=\alpha_{m}-\alpha_{f}$. The reason is that for a solution $(y(t), z(t), a(t))$ and values $\left(y_{0}, z_{0}\right)$ satisfying $y_{0}-y\left(t_{0}\right)=$ $O\left(h^{2}\right), z_{0}-z\left(t_{0}\right)=O\left(h^{2}\right)$, we have 


$$
a_{1+\alpha}-a\left(t_{1+\alpha}\right)=O\left(h^{2}\right) \quad \text { when } \quad a_{\alpha}-a\left(t_{\alpha}\right)=O\left(h^{2}\right)
$$

whereas we only have $a_{1+\alpha}-a\left(t_{1}\right)=O(h)$ for $\alpha \neq 0$ and when $a_{\alpha}-a\left(t_{0}\right)=$ $O\left(h^{2}\right)$ or $a_{\alpha}-a\left(t_{\alpha}\right)=O\left(h^{2}\right)$. This can be seen as follows. We rewrite (2c) as

$$
\left(1-\alpha_{m}\right) a_{1+\alpha}+\alpha_{m} a_{\alpha}=\left(1-\alpha_{f}\right) M^{-1} f\left(t_{1}, y_{1}, z_{1}\right)+\alpha_{f} M^{-1} f\left(t_{0}, y_{0}, z_{0}\right) .
$$

Since $a(t)=M^{-1} f(t, y(t), z(t)), y_{1}-y\left(t_{1}\right)=O\left(h^{2}\right)$, and $z_{1}-z\left(t_{1}\right)=O\left(h^{2}\right)$ we have

$$
M^{-1} f\left(t_{1}, y_{1}, z_{1}\right)=a\left(t_{0}\right)+h a^{\prime}\left(t_{0}\right)+O\left(h^{2}\right), M^{-1} f\left(t_{0}, y_{0}, z_{0}\right)=a\left(t_{0}\right)+O\left(h^{2}\right)
$$

Hence, for the right-hand side of (4) we obtain

$$
\begin{array}{rl}
\left(1-\alpha_{f}\right) M^{-1} f\left(t_{1}, y_{1}, z_{1}\right)+\alpha_{f} M^{-1} & f\left(t_{0}, y_{0}, z_{0}\right) \\
& =a\left(t_{0}\right)+h\left(1-\alpha_{f}\right) a^{\prime}\left(t_{0}\right)+O\left(h^{2}\right) .
\end{array}
$$

Since

$a\left(t_{1+\alpha}\right)=a\left(t_{0}\right)+h(1+\alpha) a^{\prime}\left(t_{0}\right)+O\left(h^{2}\right), \quad a\left(t_{\alpha}\right)=a\left(t_{0}\right)+h \alpha a^{\prime}\left(t_{0}\right)+O\left(h^{2}\right)$, we have

$$
\left(1-\alpha_{m}\right) a\left(t_{1+\alpha}\right)+\alpha_{m} a\left(t_{\alpha}\right)=a\left(t_{0}\right)+h\left(1-\alpha_{m}+\alpha\right) a^{\prime}\left(t_{0}\right)+O\left(h^{2}\right) .
$$

Thus, from (4-6), we obtain

$$
\begin{aligned}
\left(1-\alpha_{m}\right)\left(a_{1+\alpha}-a\left(t_{1+\alpha}\right)\right)+\alpha_{m}( & \left.a_{\alpha}-a\left(t_{\alpha}\right)\right) \\
& =h\left(-\alpha_{f}+\alpha_{m}-\alpha\right) a^{\prime}\left(t_{0}\right)+O\left(h^{2}\right) .
\end{aligned}
$$

Hence, (3) is satisfied for $\alpha=\alpha_{m}-\alpha_{f}$.

\section{Choosing the Initial Value of $a_{\alpha}$ for the First Integration Step}

Here we give two possible choices for the initial value of $a_{\alpha}$ to be used for the first integration step. For $\alpha_{m}=0$, for example for the HHT- $\alpha$ method, we see from (7) that taking $a_{\alpha}:=a_{0}$ where $M a_{0}=f\left(t_{0}, y_{0}, z_{0}\right)$ still leads to the estimate $a_{1+\alpha}-a\left(t_{1+\alpha}\right)=O\left(h^{2}\right)$. For $\alpha_{m} \neq 0$ it is better to define $a_{\alpha}$ such that $a_{\alpha}-a\left(t_{\alpha}\right)=O\left(h^{2}\right)$, for example implicitly by

$$
M a_{\alpha}=(1-\alpha) f\left(t_{0}, y_{0}, z_{0}\right)+\alpha f\left(t_{1}, y_{1}, z_{1}\right)
$$

as proposed by Lunk and Simeon [7]. Nevertheless, taking $a_{\alpha}:=a_{0}$ in fact does not affect the order of global convergence of the $y$ and $z$ components, see Theorem 1 in Section 6 . 


\section{Nonconstant Mass Matrix $M(t, y)$}

We consider $M(t, y) y^{\prime \prime}=f\left(t, y, y^{\prime}\right)$ where $M(t, y)$ is a nonconstant mass matrix assumed to be nonsingular. These equations are equivalent to the semiexplicit system of index 1 DAEs

$$
y^{\prime}=z, \quad z^{\prime}=a, \quad 0=M(t, y) a-f(t, y, z) .
$$

A natural extension of the generalized $-\alpha$ method of (2) is to replace (2c) with

$$
\left(1-\alpha_{m}\right) M_{1+\alpha} a_{1+\alpha}+\alpha_{m} M_{\alpha} a_{\alpha}=\left(1-\alpha_{f}\right) f\left(t_{1}, y_{1}, z_{1}\right)+\alpha_{f} f\left(t_{0}, y_{0}, z_{0}\right)
$$

where

$$
M_{1+\alpha} \approx M\left(t_{1+\alpha}, y\left(t_{1+\alpha}\right)\right), \quad M_{\alpha} \approx M\left(t_{\alpha}, y\left(t_{\alpha}\right)\right) .
$$

For example we can take explicitly

$M_{1+\alpha}:=M\left(t_{1+\alpha}, y_{0}+h(1+\alpha) z_{0}\right), \quad M_{\alpha}:=M_{(1+\alpha)-1}$ or $M\left(t_{\alpha}, y_{0}+h \alpha z_{0}\right)$

where $M_{(1+\alpha)-1}$ denotes the matrix $M_{1+\alpha}$ used at the previous time-step. Second order of convergence is a consequence of Theorem 1 in Section 6 .

\section{Holonomic Constraints $g(t, y)=0$}

We extend now the generalized $-\alpha$ method to systems having holonomic constraints $g(t, y)=0$. More precisely we consider

$$
M(t, y) y^{\prime \prime}=f\left(t, y, y^{\prime}, \lambda\right), \quad 0=g(t, y) .
$$

Using the notation $g_{y}(t, y):=\frac{\partial g}{\partial y}(t, y)$, usually $f\left(t, y, y^{\prime}, \lambda\right)=f_{0}\left(t, y, y^{\prime}\right)-$ $g_{y}^{T}(t, y) \lambda$ and the term $-g_{y}^{T}(t, y) \lambda$ containing algebraic variables $\lambda$ represents reaction forces due to the holonomic constraints $g(t, y)=0$. Differentiating $0=g(t, y)$ once with respect to $t$ we obtain

$$
0=(g(t, y))^{\prime}=g_{t}(t, y)+g_{y}(t, y) y^{\prime} .
$$

Hence, we consider overdetermined systems of index 2 differential-algebraic equations (ODAEs) of the form

$$
\begin{aligned}
& y^{\prime}=z, \quad z^{\prime}=a, \quad 0=M(t, y) a-f(t, y, z, \lambda), \\
& 0=g(t, y), \quad 0=g_{t}(t, y)+g_{y}(t, y) z
\end{aligned}
$$

where we assume the matrix

$$
\left(\begin{array}{lc}
M(t, y) & -f_{\lambda}(t, y, z, \lambda) \\
g_{y}(t, y) & O
\end{array}\right) \text { is nonsingular. }
$$


When $f(t, y, z, \lambda)=f_{0}(t, y, z)-g_{y}^{T}(t, y) \lambda$, this matrix becomes

$$
\left(\begin{array}{ll}
M(t, y) & g_{y}^{T}(t, y) \\
g_{y}(t, y) & O
\end{array}\right)
$$

and it is symmetric when $M(t, y)$ is symmetric. At $t_{0}$ we consider consistent initial conditions $\left(y_{0}, z_{0}, a_{0}, \lambda_{0}\right)$, i.e.,

$$
\begin{aligned}
& 0=M\left(t_{0}, y_{0}\right) a_{0}-f\left(t_{0}, y_{0}, z_{0}, \lambda_{0}\right), \\
& 0=g\left(t_{0}, y_{0}\right), \\
& 0=g_{t}\left(t_{0}, y_{0}\right)+g_{y}\left(t_{0}, y_{0}\right) z_{0}, \\
& 0=g_{t t}\left(t_{0}, y_{0}\right)+2 g_{t y}\left(t_{0}, y_{0}\right) z_{0}+g_{y y}\left(t_{0}, y_{0}\right)\left(z_{0}, z_{0}\right)+g_{y}\left(t_{0}, y_{0}\right) a_{0} .
\end{aligned}
$$

Several extensions of the HHT- $\alpha$ method have been proposed. Cardona and Géradin [1] analyze a direct extension of the HHT- $\alpha$ method to linear index 3 DAEs. They show that a direct application of the HHT- $\alpha$ method is inconsistent and suffers from instabilities. Yen, Petzold, and Raha [11] propose a first order extension of the HHT- $\alpha$ method based on projecting the solution of the underlying ODEs onto the constraints (including the index 1 acceleration level constraints) after each step. More recently, second order extensions of the HHT- $\alpha$ method and generalized- $\alpha$ method have been proposed independently by Jay and Negrut [5] and by Lunk and Simeon [7] assuming additivity of $f(t, y, z, \lambda)=f_{0}(t, y, z)+f_{1}(t, y, \lambda)$. Here, we propose a different extension of the generalized $-\alpha$ method without making this assumption:

$$
\begin{aligned}
y_{1} & =y_{0}+h z_{0}+\frac{h^{2}}{2}\left((1-2 \beta) a_{\alpha}+2 \beta \widetilde{a}_{1+\alpha}\right) \\
z_{1} & =z_{0}+h\left((1-\gamma) a_{\alpha}+\gamma a_{1+\alpha}\right), \\
\left(1-\alpha_{m}\right) M_{1+\alpha} \widetilde{a}_{1+\alpha}+\alpha_{m} M_{\alpha} a_{\alpha} & =\left(1-\alpha_{f}\right) f\left(t_{1}, y_{1}, z_{1}, \widetilde{\lambda}_{1}\right) \\
& +\alpha_{f} f\left(t_{0}, y_{0}, z_{0}, \lambda_{0}\right), \\
\left(1-\alpha_{m}\right) M_{1+\alpha} a_{1+\alpha}+\alpha_{m} M_{\alpha} a_{\alpha} & =\left(1-\alpha_{f}\right) f\left(t_{1}, y_{1}, z_{1}, \lambda_{1}\right) \\
& +\alpha_{f} f\left(t_{0}, y_{0}, z_{0}, \lambda_{0}\right), \\
0 & =g\left(t_{1}, y_{1}\right), \\
0 & =g_{t}\left(t_{1}, y_{1}\right)+g_{y}\left(t_{1}, y_{1}\right) z_{1} .
\end{aligned}
$$

When $f(t, y, z, \lambda)=f_{0}(t, y, z)-g_{y}^{T}(t, y) \lambda$ we can replace (9c) by

$$
\left(1-\alpha_{m}\right) M_{1+\alpha}\left(\widetilde{a}_{1+\alpha}-a_{1+\alpha}\right)=\left(1-\alpha_{f}\right) g_{y}^{T}\left(t_{1}, y_{1}\right)\left(\lambda_{1}-\widetilde{\lambda}_{1}\right) .
$$

Second order of convergence is a consequence of Theorem 1 in Section 6 .

\section{Nonholonomic Constraints $k\left(t, y, y^{\prime}\right)=0$}

We extend now the generalized- $\alpha$ method to systems having nonholonomic constraints $k\left(t, y, y^{\prime}\right)=0$. More precisely we consider 


$$
M(t, y) y^{\prime \prime}=f\left(t, y, y^{\prime}, \psi\right), \quad 0=k\left(t, y, y^{\prime}\right) .
$$

Usually $f\left(t, y, y^{\prime}, \psi\right)=f_{0}\left(t, y, y^{\prime}\right)-k_{y^{\prime}}^{T}\left(t, y, y^{\prime}\right) \psi$ and the term $-k_{y^{\prime}}^{T}\left(t, y, y^{\prime}\right) \psi$ containing algebraic variables $\psi$ represents reaction forces due to the nonholonomic constraints $k\left(t, y, y^{\prime}\right)=0$. Hence, we consider systems of index 2 DAEs of the form

$$
y^{\prime}=z, \quad z^{\prime}=a, \quad 0=M(t, y) a-f(t, y, z, \psi), \quad 0=k(t, y, z),
$$

and we assume the matrix

$$
\left(\begin{array}{cc}
M(t, y) & -f_{\psi}(t, y, z, \psi) \\
k_{z}(t, y, z) & O
\end{array}\right) \text { is nonsingular } .
$$

When $f(t, y, z, \psi)=f_{0}(t, y, z)-k_{z}^{T}(t, y, z) \psi$, this matrix becomes

$$
\left(\begin{array}{cc}
M(t, y) & k_{z}^{T}(t, y, z) \\
k_{z}(t, y, z) & O
\end{array}\right)
$$

and it is symmetric when $M(t, y)$ is symmetric. At $t_{0}$ we consider consistent initial conditions $\left(y_{0}, z_{0}, a_{0}, \psi_{0}\right)$, i.e.,

$$
\begin{aligned}
& 0=M\left(t_{0}, y_{0}\right) a_{0}-f\left(t_{0}, y_{0}, z_{0}, \psi_{0}\right) \\
& 0=k\left(t_{0}, y_{0}, z_{0}\right), \\
& 0=k_{t}\left(t_{0}, y_{0}, z_{0}\right)+k_{y}\left(t_{0}, y_{0}, z_{0}\right) z_{0}+k_{z}\left(t_{0}, y_{0}, z_{0}\right) a_{0} .
\end{aligned}
$$

We propose the following extension of the generalized $-\alpha$ method

$$
\begin{aligned}
y_{1} & =y_{0}+h z_{0}+\frac{h^{2}}{2}\left((1-2 \beta) a_{\alpha}+2 \beta a_{1+\alpha}\right), \\
z_{1} & =z_{0}+h\left((1-\gamma) a_{\alpha}+\gamma a_{1+\alpha}\right), \\
\left(1-\alpha_{m}\right) M_{1+\alpha} a_{1+\alpha}+\alpha_{m} M_{\alpha} a_{\alpha} & =\left(1-\alpha_{f}\right) f\left(t_{1}, y_{1}, z_{1}, \psi_{1}\right) \\
0 & =k\left(t_{1}, y_{1}, z_{1}\right) .
\end{aligned}
$$

Second order of convergence is a consequence of Theorem 1 in Section 6 .

\section{General Extension and Convergence}

We extend now the generalized- $\alpha$ method to systems having a nonconstant mass matrix $M(t, y)$, holonomic constraints $g(t, y)=0$, and nonholonomic constraints $k\left(t, y, y^{\prime}\right)=0$. The algebraic variables $\lambda$ are associated with the holonomic constraints $g(t, y)=0$ and $g_{t}(t, y)+g_{y}(t, y) y^{\prime}=0$ which results from differentiating $g(t, y)=0$ with respect to $t$. The algebraic variables $\psi$ are associated with the nonholonomic constraints $k\left(t, y, y^{\prime}\right)=0$. Hence, we 
consider overdetermined systems of index 2 differential-algebraic equations (ODAEs) of the form

$$
\begin{aligned}
y^{\prime} & =z, \\
M(t, y) z^{\prime} & =f(t, y, z, \lambda, \psi), \\
0 & =g(t, y), \\
0 & =g_{t}(t, y)+g_{y}(t, y) z, \\
0 & =k(t, y, z),
\end{aligned}
$$

and we assume the matrix

$$
\left(\begin{array}{ccc}
M(t, y) & -f_{\lambda}(t, y, z, \lambda, \psi)-f_{\psi}(t, y, z, \lambda, \psi) \\
g_{y}(t, y) & O & O \\
k_{z}(t, y, z) & O & O
\end{array}\right) \text { is nonsingular }
$$

When $f(t, y, z, \lambda, \psi)=f_{0}(t, y, z)-g_{y}^{T}(t, y) \lambda-k_{z}^{T}(t, y, z) \psi$, this matrix becomes

$$
\left(\begin{array}{ccc}
M(t, y) & g_{y}^{T}(t, y) & k_{z}^{T}(t, y, z) \\
g_{y}(t, y) & O & O \\
k_{z}(t, y, z) & O & O
\end{array}\right)
$$

and it is symmetric when $M(t, y)$ is symmetric. At $t_{0}$ we consider consistent initial conditions $\left(y_{0}, z_{0}, a_{0}, \lambda_{0}, \psi_{0}\right)$, i.e.,

$$
\begin{aligned}
& 0=M\left(t_{0}, y_{0}\right) a_{0}-f\left(t_{0}, y_{0}, z_{0}, \lambda_{0}, \psi_{0}\right) \\
& 0=g\left(t_{0}, y_{0}\right) \\
& 0=g_{t}\left(t_{0}, y_{0}\right)+g_{y}\left(t_{0}, y_{0}\right) z_{0} \\
& 0=k\left(t_{0}, y_{0}, z_{0}\right) \\
& 0=g_{t t}\left(t_{0}, y_{0}\right)+2 g_{t y}\left(t_{0}, y_{0}\right) z_{0}+g_{y y}\left(t_{0}, y_{0}\right)\left(z_{0}, z_{0}\right)+g_{y}\left(t_{0}, y_{0}\right) a_{0}, \\
& 0=k_{t}\left(t_{0}, y_{0}, z_{0}\right)+k_{y}\left(t_{0}, y_{0}, z_{0}\right) z_{0}+k_{z}\left(t_{0}, y_{0}, z_{0}\right) a_{0} .
\end{aligned}
$$

We propose an extension of the generalized- $\alpha$ method which does not use any additive structure of $f(t, y, z, \lambda, \psi)$. We call it the generalized- $\alpha$-SOI2 method (SOI2 stands for Stabilized Overdetermined Index 2). One step $\left(t_{0}, y_{0}, z_{0}, a_{\alpha}, \lambda_{0}, \psi_{0}\right) \mapsto\left(t_{1}, y_{1}, z_{1}, a_{1+\alpha}, \lambda_{1}, \psi_{1}\right)$ with step-size $h$ of the generalized- $\alpha-$ SOI2 method for (11) can be expressed as follows 


$$
\begin{aligned}
y_{1} & =y_{0}+h z_{0}+\frac{h^{2}}{2}\left((1-2 \beta) a_{\alpha}+2 \beta \widetilde{a}_{1+\alpha}\right) \\
\widetilde{z}_{1} & =z_{0}+h\left((1-\gamma) a_{\alpha}+\gamma \widetilde{a}_{1+\alpha}\right) \\
z_{1} & =z_{0}+h\left((1-\gamma) a_{\alpha}+\gamma a_{1+\alpha}\right) \\
\left(1-\alpha_{m}\right) M_{1+\alpha} \widetilde{a}_{1+\alpha}+\alpha_{m} M_{\alpha} a_{\alpha} & =\left(1-\alpha_{f}\right) f\left(t_{1}, y_{1}, z_{1}, \widetilde{\lambda}_{1}, \widetilde{\psi}_{1}\right) \\
& +\alpha_{f} f\left(t_{0}, y_{0}, z_{0}, \lambda_{0}, \psi_{0}\right) \\
\left(1-\alpha_{m}\right) M_{1+\alpha} a_{1+\alpha}+\alpha_{m} M_{\alpha} a_{\alpha} & =\left(1-\alpha_{f}\right) f\left(t_{1}, y_{1}, z_{1}, \lambda_{1}, \psi_{1}\right) \\
0 & =g\left(t_{1}, y_{1}\right), \quad+\alpha_{f} f\left(t_{0}, y_{0}, z_{0}, \lambda_{0}, \psi_{0}\right) \\
0 & =g_{t}\left(t_{1}, y_{1}\right)+g_{y}\left(t_{1}, y_{1}\right) z_{1} \\
0 & =k\left(t_{1}, y_{1}, \widetilde{z}_{1}\right) \\
0 & =k\left(t_{1}, y_{1}, z_{1}\right)
\end{aligned}
$$

where $M_{1+\alpha}:=M\left(t_{1+\alpha}, y_{0}+h(1+\alpha) z_{0}\right)$ and $M_{\alpha}:=M_{(1+\alpha)-1}$ or $M\left(t_{\alpha}, y_{0}+\right.$ $\left.h \alpha z_{0}\right)$. The auxiliary variables $\widetilde{z}_{1}, \widetilde{a}_{1+\alpha}, \widetilde{\lambda}_{1}, \widetilde{\psi}_{1}$ are just local to the current step, they are not propagated. The possibility to replace $\widetilde{\psi}_{1}$ by $\psi_{1}$ in (13d) and to suppress the equations (13b)-(13h) and the auxiliary variables $\widetilde{z}_{1}, \widetilde{\psi}_{1}$ remains to be investigated. When $f(t, y, z, \lambda, \psi)=f_{0}(t, y, z)-g_{y}^{T}(t, y) \lambda-k_{z}^{T}(t, y, z) \psi$ we can replace $(13 \mathrm{~d})$ by

$$
\begin{aligned}
\left(1-\alpha_{m}\right) M_{1+\alpha}\left(\widetilde{a}_{1+\alpha}-a_{1+\alpha}\right)=\left(1-\alpha_{f}\right) & g_{y}^{T}\left(t_{1}, y_{1}\right)\left(\lambda_{1}-\widetilde{\lambda}_{1}\right) \\
& +\left(1-\alpha_{f}\right) k_{z}^{T}\left(t_{1}, y_{1}, z_{1}\right)\left(\psi_{1}-\widetilde{\psi}_{1}\right) .
\end{aligned}
$$

Convergence analysis of the generalized- $\alpha-$ SOI2 method is not straightforward. We have the following convergence result:

Theorem 1. Consider the overdetermined system of DAEs (11) under the assumption (12) with consistent initial conditions $\left(y_{0}, z_{0}, a_{0}, \lambda_{0}, \psi_{0}\right)$ at $t_{0}$ and exact solution $(y(t), z(t), a(t), \lambda(t), \psi(t))$. Suppose that $a_{\alpha}-a\left(t_{0}+\alpha h\right)=$ $O(h)$ (e.g., $\left.a_{\alpha}:=a_{0}\right), \alpha_{m} \neq 1, \alpha_{f} \neq 1, \beta \neq 0, \gamma \neq 0$, and $r<1$ where $r:=\left|\alpha_{m} /\left(1-\alpha_{m}\right)\right|$. Then the generalized- $\alpha-S O I 2$ numerical approximation $\left(y_{n}, z_{n}, a_{n+\alpha}, \lambda_{n}, \psi_{n}\right)$, see (13), satisfies for $0 \leq h \leq h_{\max }$ and $t_{n}-t_{0}=n h \leq$ Const, the following global error estimates

$$
\begin{aligned}
& y_{n}-y\left(t_{n}\right)=O\left(h^{2}\right), \quad z_{n}-z\left(t_{n}\right)=O\left(h^{2}\right), \\
& a_{n+\alpha}-a\left(t_{n}+\alpha h\right)=O\left(h^{2}+r^{n} \delta_{0}\right), \\
& \lambda_{n}-\lambda\left(t_{n}\right)=O\left(h^{2}+r^{n} \delta_{0}\right), \quad \psi_{n}-\psi\left(t_{n}\right)=O\left(h^{2}+r^{n} \delta_{0}\right)
\end{aligned}
$$

where $\delta_{0}:=\left\|a_{\alpha}-a\left(t_{0}+\alpha\right)\right\|=O(h)$. If $\alpha_{m}=0$ or $a_{\alpha}-a\left(t_{0}+\alpha h\right)=O\left(h^{2}\right)$ we have

$$
a_{n+\alpha}-a\left(t_{n}+\alpha h\right)=O\left(h^{2}\right), \quad \lambda_{n}-\lambda\left(t_{n}\right)=O\left(h^{2}\right), \quad \psi_{n}-\psi\left(t_{n}\right)=O\left(h^{2}\right) .
$$


Theorem 1 remains valid for variable step-sizes, see Section 7. A proof of this theorem will be given in a forthcoming paper [6]. It is long and technical and is thus omitted here.

\section{Adjustments for Variable Step-Sizes $h_{n}$}

When applying the generalized- $\alpha$ method with variable step-sizes, the values $a_{n+\alpha}$ and $M_{n+\alpha} a_{n+\alpha}$ must be adjusted before each new step in order to preserve the second order of the method for all components. Consider a previous step starting at $t_{n-1}$ with step-size $h_{n-1}$ and a new step starting at $t_{n}=t_{n-1}+h_{n-1}$ with step-size $h_{n}$. The value $a_{n-1+\alpha}$ used in the previous step is an approximation of $a(t)$ at $t_{n-1}+\alpha h_{n-1}$ i.e., $a_{n-1+\alpha} \approx a\left(t_{n-1}+\alpha h_{n-1}\right)$. The value $a_{n+\alpha}$ obtained in the previous step is an approximation of $a(t)$ at $t_{n-1}+(1+\alpha) h_{n-1}=t_{n}+\alpha h_{n-1}$ i.e., $a_{n+\alpha} \approx a\left(t_{n}+\alpha h_{n-1}\right)$. For the current time-step starting at $t_{n}$ with step-size $h_{n}$ we need the value $a_{n+\alpha}$ to be an approximation of $a(t)$ at $t_{n}+\alpha h_{n}$, i.e., $a_{n+\alpha} \approx a\left(t_{n}+\alpha h_{n}\right)$. By linearly interpolating $a_{n-1+\alpha}$ at $t_{n-1}+\alpha h_{n-1}$ and $a_{n+\alpha}$ at $t_{n}+\alpha h_{n-1}$ and by extrapolating at $t_{n}+\alpha h_{n}, a_{n+\alpha}$ can be replaced by

$$
a_{n+\alpha}:=a_{n+\alpha}+\alpha\left(\frac{h_{n}}{h_{n-1}}-1\right)\left(a_{n+\alpha}-a_{n-1+\alpha}\right) .
$$

Analogously we can replace $M_{n+\alpha} a_{n+\alpha}$ by

$$
\begin{aligned}
M_{n+\alpha} a_{n+\alpha}:= & M_{n+\alpha} a_{n+\alpha} \\
& +\alpha\left(\frac{h_{n}}{h_{n-1}}-1\right)\left(M_{n+\alpha} a_{n+\alpha}-M_{n-1+\alpha} a_{n-1+\alpha}\right) .
\end{aligned}
$$

These adjusting formulas (14) have several advantages:

- They are simple to implement;

- Their computational cost is almost negligible;

- They are valid for ODEs and DAEs;

- They preserve second order of convergence.

These modifications are not necessary to preserve the second order of convergence for the $y$ and $z$ components. However, they are recommended since their computational cost is almost negligible and they allow second order of convergence for the components $a, \lambda$, and $\psi$.

\section{Numerical Experiments}

\subsection{A Nonlinear Mathematical Test Problem}

To illustrate Theorem 1 numerically we first consider the following nonlinear mathematical test problem 


$$
\begin{aligned}
& \left(\begin{array}{l}
y_{1}^{\prime} \\
y_{2}^{\prime}
\end{array}\right)=\left(\begin{array}{l}
z_{1} \\
z_{2}
\end{array}\right) \\
& \left(\begin{array}{cc}
y_{1} & y_{2}-e^{-2 t} \\
\sin \left(y_{1}-e^{t}\right) & y_{1} y_{2}
\end{array}\right)\left(\begin{array}{l}
z_{1}^{\prime} \\
z_{2}^{\prime}
\end{array}\right)= \\
& \left(\begin{array}{c}
e^{t}\left(y_{1} z_{2}+2 y_{2} z_{1}\right)+e^{2 t} y_{1} \lambda_{1}-y_{1} z_{2} \psi_{1}-2 \\
e^{-t}\left(0.5 y_{2} z_{2}-2 y_{1} z_{1} y_{2} z_{2}+y_{2} \lambda_{1}^{2}\right)-y_{1} y_{2} z_{1} \psi_{1}^{3}+e^{3 t}
\end{array}\right) \\
& 0=g(t, y)=y_{1}^{2} y_{2}-1, \\
& 0=g_{t}(t, y)+g_{y}(t, y) z=2 y_{1} y_{2} z_{1}+y_{1}^{2} z_{2}, \\
& 0=k(t, y, z)=y_{1} z_{1} z_{2}+2 \text {. }
\end{aligned}
$$

Observe that this problem is nonlinear in the algebraic variables $\lambda_{1}$ and $\psi_{1}$. The following initial conditions at $t_{0}=0$ have been used: $y_{1}(0)=1, y_{2}(0)=1$, $z_{1}(0)=1, z_{2}(0)=-2, \lambda_{1}(0)=1, \psi_{1}(0)=1$. The exact solution is given explicitly as follows: $y_{1}(t)=e^{t}, y_{2}(t)=e^{-2 t}, z_{1}(t)=e^{t}, z_{2}(t)=-2 e^{-2 t}$, $\lambda_{1}(t)=e^{-t}, \psi_{1}(t)=e^{t}$. We have applied the generalized $-\alpha-$ SOI2 method, see (13), with damping parameter $\rho_{\infty}=0.2$ and variable step-sizes alternating between $h / 3$ and $2 h / 3$ for various values of $h$. Using the adjusting formulas (14) for $a_{n+\alpha}$ and $M_{n+\alpha} a_{n+\alpha}$ we observe global convergence of order 2 at $t_{n}=1$ in Fig. 1. Without these modifications a reduction of the order of convergence to 1 for the components $a, \lambda$, and $\psi$ can be observed in Fig. 2 .

\subsection{A Pendulum Model}

As a second numerical experiment we consider the pendulum model in Fig. 3 where we denote $y_{1}:=x, y_{2}:=y, y_{3}:=\theta$. The constrained equations of motion associated with this model are

$$
\begin{gathered}
\left(\begin{array}{l}
y_{1}^{\prime} \\
y_{2}^{\prime} \\
y_{3}^{\prime}
\end{array}\right)=\left(\begin{array}{l}
z_{1} \\
z_{2} \\
z_{3}
\end{array}\right) \\
\left(\begin{array}{lll}
m & 0 & 0 \\
0 & m & 0 \\
0 & 0 & m \frac{L^{2}}{3}
\end{array}\right)\left(\begin{array}{l}
z_{1}^{\prime} \\
z_{2}^{\prime} \\
z_{3}^{\prime}
\end{array}\right)=\left(\begin{array}{c}
0 \\
-m g \\
-c z_{3}-k \cdot\left(y_{3}-\frac{3 \pi}{2}\right)
\end{array}\right) \\
-\left(\begin{array}{cc}
1 & 0 \\
0 & 1 \\
L \sin \left(y_{3}\right)-L \cos \left(y_{3}\right)
\end{array}\right)\left(\begin{array}{c}
\lambda_{1} \\
\lambda_{2}
\end{array}\right), \\
\left(\begin{array}{l}
0 \\
0
\end{array}\right)=g(t, y)=\left(\begin{array}{l}
y_{1}-L \cos \left(y_{3}\right) \\
y_{2}-L \sin \left(y_{3}\right)
\end{array}\right) \\
\left(\begin{array}{l}
0 \\
0
\end{array}\right)=g_{t}(t, y)+g_{y}(t, y) z=\left(\begin{array}{c}
z_{1}+L \sin \left(y_{3}\right) z_{3} \\
z_{2}-L \cos \left(y_{3}\right) z_{3}
\end{array}\right) .
\end{gathered}
$$

The pendulum is started from consistent initial conditions corresponding to $y_{3}(0)=3 \pi / 2, z_{3}(0)=10$. The parameters used are given in the caption 


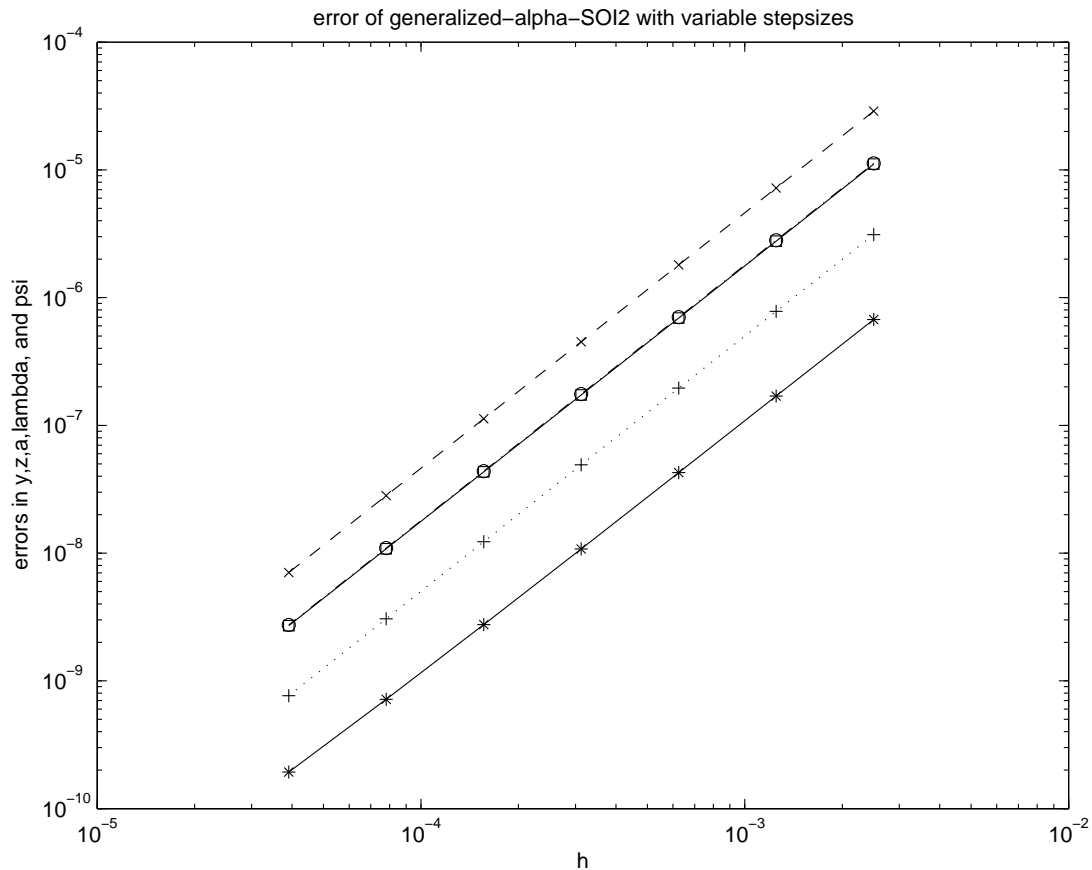

Fig. 1. Global errors $\left\|y_{n}-y\left(t_{n}\right)\right\|_{2}(\square),\left\|z_{n}-z\left(t_{n}\right)\right\|_{2}(\circ),\left\|a_{n+\alpha}-a\left(t_{n}+\alpha h\right)\right\|_{2}(\times)$, $\left\|\lambda_{n}-\lambda\left(t_{n}\right)\right\|_{2}(+),\left\|\psi_{n}-\psi\left(t_{n}\right)\right\|_{2}(*)$ of the generalized- $\alpha$-SOI2 method $\left(\rho_{\infty}=0.2\right)$ at $t_{n}=1$ for the test problem (15) with variable step-sizes alternating between $h / 3$ and $2 h / 3$ using the adjusting formulas (14) for $a_{n+\alpha}$ and $M_{n+\alpha} a_{n+\alpha}$

of Fig. 3. We have applied the generalized- $\alpha-$ SOI2 method, see (13), with damping parameter $\rho_{\infty}=0.2$ and variable step-sizes alternating between $h / 3$ and $2 h / 3$ for various values of $h$. Using the adjusting formula (14a) for $a_{n+\alpha}$ we observe global convergence of order 2 at $t_{n}=2$ in Fig. 4.

\section{$8.3 \alpha_{m}=0$ and Holonomic Constraints: HHT-SOI2}

As mentioned in Section 1 the generalized $-\alpha$ method for $\alpha_{m}=0$ corresponds to the HHT $-\alpha$ method. In this section we only consider the generalized $-\alpha-$ SOI2 method for $\alpha_{m}=0$ and systems for which

- The constraints are holonomic $0=g(t, y)$;

- Forces are of the form $f(t, y, z, \lambda)=f_{0}(t, y, z)-g_{y}^{T}(t, y) \lambda$.

Note that these two conditions are satisfied by a vast number of multibody systems. In this situation, denoting $\alpha:=-\alpha_{f}, \widetilde{\delta}:=\widetilde{a}_{1+\alpha}-a_{1+\alpha}$, and $\widetilde{\mu}:=$ $(1+\alpha)\left(\lambda_{1}-\widetilde{\lambda}_{1}\right)$, the generalized- $\alpha-$ SOI2 method given in (9) becomes 


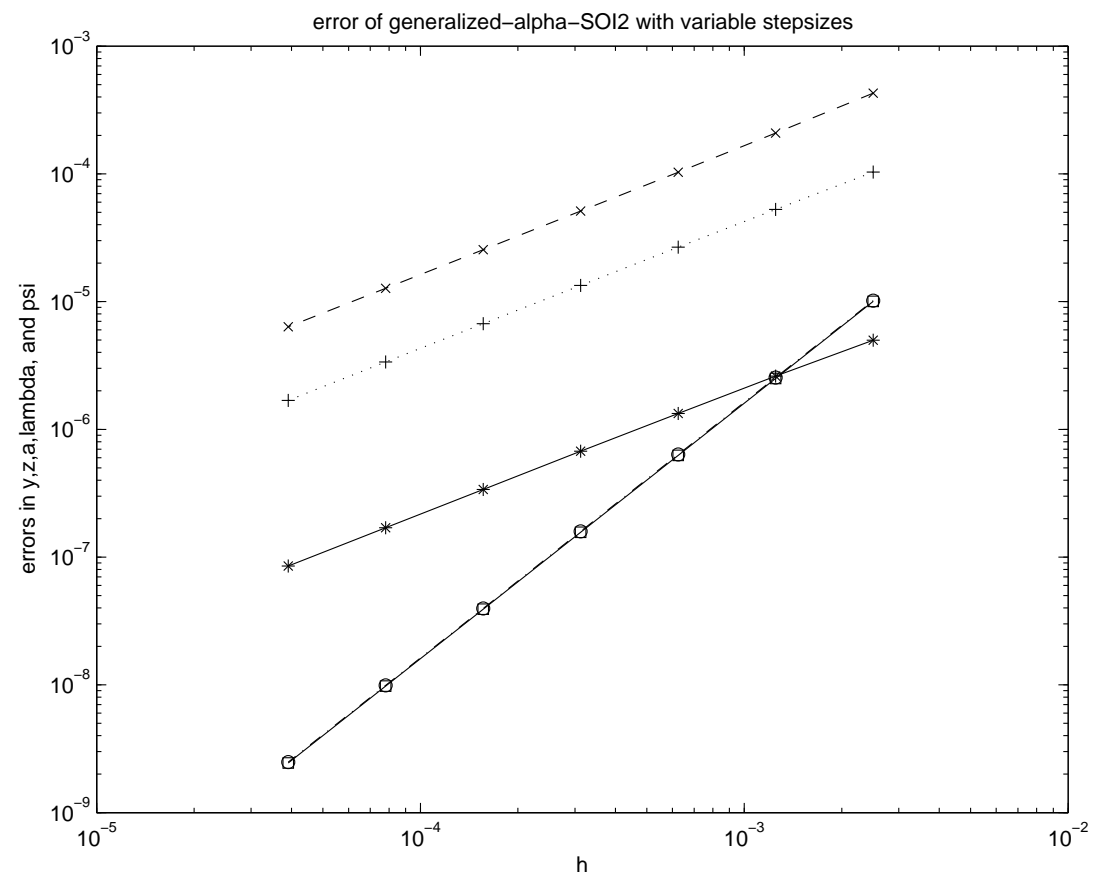

Fig. 2. Global errors $\left\|y_{n}-y\left(t_{n}\right)\right\|_{2}(\square),\left\|z_{n}-z\left(t_{n}\right)\right\|_{2}(\circ),\left\|a_{n+\alpha}-a\left(t_{n}+\alpha h\right)\right\|_{2}(\times)$, $\left\|\lambda_{n}-\lambda\left(t_{n}\right)\right\|_{2}(+),\left\|\psi_{n}-\psi\left(t_{n}\right)\right\|_{2}(*)$ of the generalized- $\alpha$-SOI2 method $\left(\rho_{\infty}=0.2\right)$ at $t_{n}=1$ for the test problem (15) with variable step-sizes alternating between $h / 3$ and $2 h / 3$ without using the adjusting formulas (14) for $a_{n+\alpha}$ and $M_{n+\alpha} a_{n+\alpha}$

$$
\begin{aligned}
y_{1} & =y_{0}+h z_{0}+\frac{h^{2}}{2}\left((1-2 \beta) a_{\alpha}+2 \beta\left(a_{1+\alpha}+\widetilde{\delta}\right)\right), \\
z_{1} & =z_{0}+h\left((1-\gamma) a_{\alpha}+\gamma a_{1+\alpha}\right) \\
M_{1+\alpha} \widetilde{\delta} & =g_{y}^{T}\left(t_{1}, y_{1}\right) \widetilde{\mu} \\
M_{1+\alpha} a_{1+\alpha} & =(1+\alpha) f\left(t_{1}, y_{1}, z_{1}, \lambda_{1}\right)-\alpha f\left(t_{0}, y_{0}, z_{0}, \lambda_{0}\right), \\
0 & =g\left(t_{1}, y_{1}\right) \\
0 & =g_{t}\left(t_{1}, y_{1}\right)+g_{y}\left(t_{1}, y_{1}\right) z_{1} .
\end{aligned}
$$

Numerical experiments have been carried out based on this method which is called HHT-SOI2 hereafter. The method HHT-SOI2 is similar in form to the HHT-I3 method proposed in [9] with the following differences:

- The velocity kinematic constraints (17f) has been added to provide constraint stabilization;

- The equation (17a) has an acceleration correction term $\widetilde{\delta}$;

- The additional equation (17c) relates the acceleration correction $\widetilde{\delta}$ to the algebraic variables $\widetilde{\mu}$ associated with the velocity kinematic constraints (17f). 


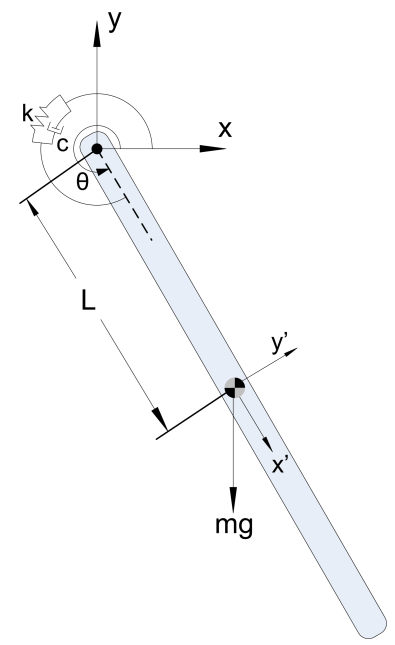

Fig. 3. A pendulum model. Parameters used (in SI units): mass $m=5$, length $L=2$, spring stiffness $k=3000$, damping coefficient $c=100$, gravitational acceleration $g=9.81$. Initial conditions used correspond to $\theta(0)=3 \pi / 2, \theta^{\prime}(0)=10$

A rigid-body slider crank model shown in Fig. 5 is used here to illustrate the velocity constraint stabilization. The equations of motion are formulated using the floating frame of reference formulation [10]. A description of this model along with initial conditions used in its analysis is provided in [8]. We have monitored the velocity of the pin connecting the crank to the ground (point $O$ in Fig. 5) using a step-size $h=2^{-10}=0.0009765625$. Ideally, the drift of the velocity constraints should be zero. When plotted in a phase plot one against the other, for the HHT-I3 integrator a limit cycle of magnitude approximately $10^{-6}$ can be observed in Fig. 6, while for the HHT-SOI2, as expected, the plot of Fig. 7 displays a collection of random points that are within machine precision.

\section{Conclusions}

The generalized $-\alpha$ method of Chung and Hulbert [2] is extended in this work to handle the case of nonlinear differential-algebraic equations associated for example with the time evolution of systems of rigid and/or flexible bodies. The proposed method, called generalized- $\alpha-$ SOI2 method, where SOI2 stands for Stabilized Overdetermined Index 2, is second order convergent for systems having nonconstant mass matrix, holonomic constraints, and/or nonholonomic constraints. For variable step-sizes, a new adjusting formula preserving the second order of the method is proposed. Numerical experiments have been carried out to verify these claims. The new extension has the same 


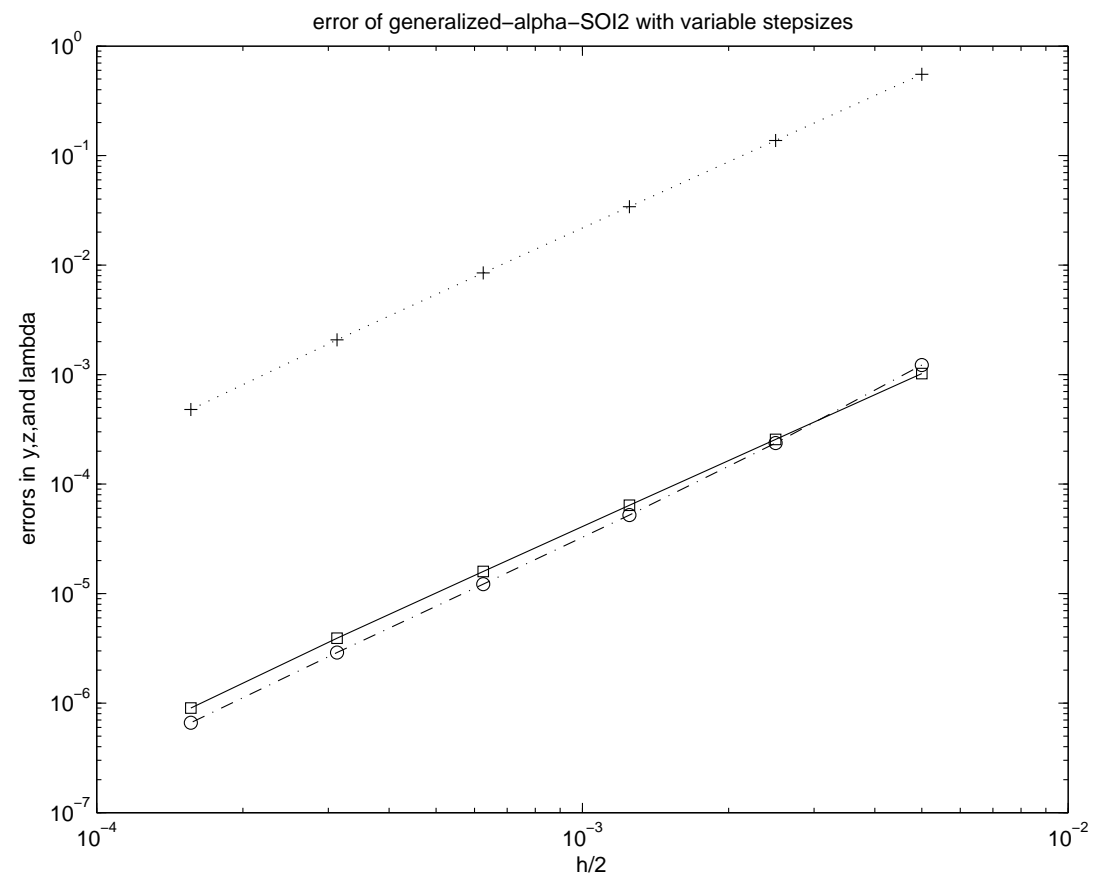

Fig. 4. Global errors $\left\|y_{n}-y\left(t_{n}\right)\right\|_{2}(\square),\left\|z_{n}-z\left(t_{n}\right)\right\|_{2}(\circ),\left\|\lambda_{n}-\lambda\left(t_{n}\right)\right\|_{2}(+)$ of the generalized- $\alpha$-SOI2 method $\left(\rho_{\infty}=0.2\right)$ at $t_{n}=2$ for the pendulum test problem (16) with variable step-size alternating between $h / 3$ and $2 h / 3$ using the adjusting formula (14a) for $a_{n+\alpha}$

user-adjustable numerical damping parameters associated with the original generalized $-\alpha$ method. Due to its semi-implicit formulation, early numerical

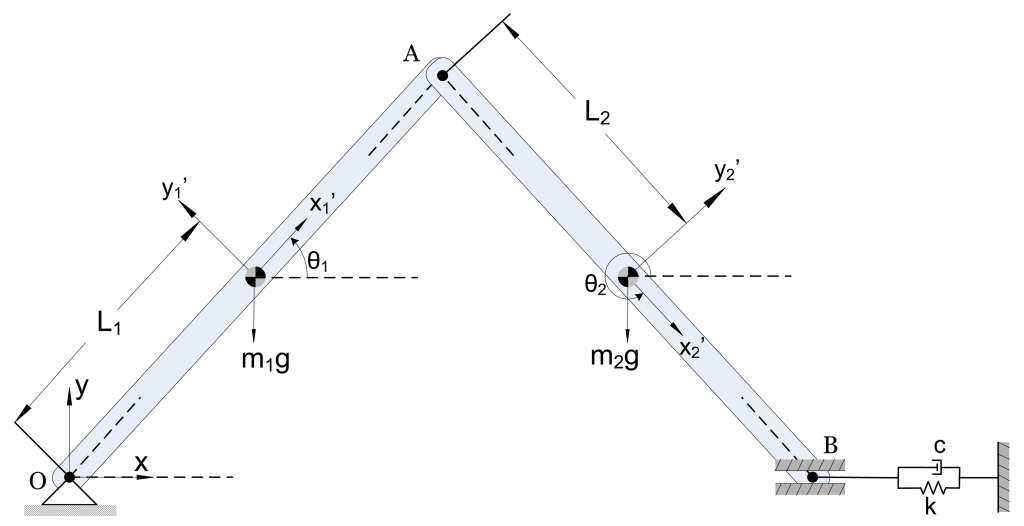

Fig. 5. Slider crank mechanism 


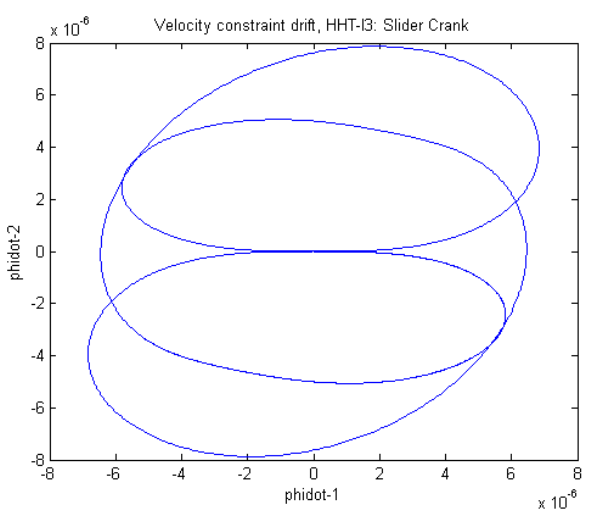

Fig. 6. Velocity kinematic constraints violation for $\mathrm{HHT}-\mathrm{I} 3$

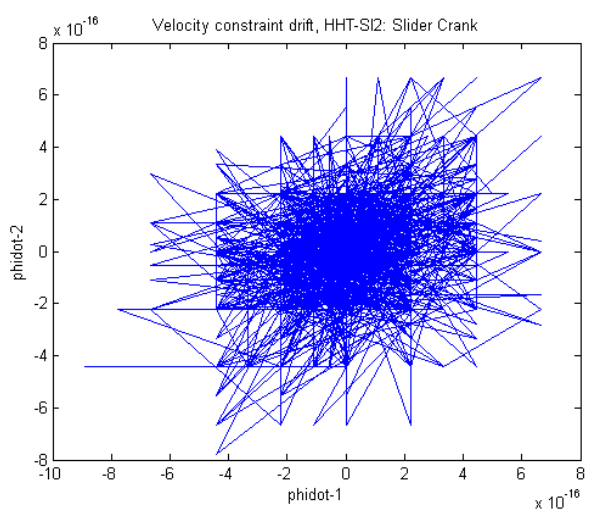

Fig. 7. Velocity kinematic constraints satisfaction for HHT-SOI2

results suggest that the new method is more efficient for large mechanical systems simulation when compared to the current state of the art in numerical integration of constrained systems in mechanics.

\section{References}

1. Cardona A, Géradin M (1989) Time integration of the equations of motion in mechanism analysis. Comput. \& Structures 33: 801-820

2. Chung J, Hulbert GM (1993) A time integration algorithm for structural dynamics with improved numerical dissipation: the generalized $-\alpha$ method. J. Appl. Mech. 60: 371-375

3. Hilber HM, Hughes TJR, Taylor RL (1977) Improved numerical dissipation for time integration algorithms in structural dynamics. Earthquake Engng Struct. Dyn. 5: 283-292

4. Hughes TJR, Taylor RL (1987) Finite element method - Linear static and dynamic finite element analysis. Prentice-Hall, Englewood Cliffs, New Jersey

5. Jay LO, Negrut D (2007) Extensions of the HHT- $\alpha$ method to differentialalgebraic equations in mechanics. ETNA 26: 190-208

6. Jay LO, Negrut D (2008) A second order extension of the generalized- $\alpha$ method for constrained systems in mechanics. Technical report, Dept. of Mathematics, Univ. of Iowa, USA. In progress

7. Lunk C, Simeon B (2006) Solving constrained mechanical systems by the family of Newmark and $\alpha$-methods. ZAMM 86: 772-784

8. Negrut D, Jay LO, Khude N, Heyn T (2007) A discussion of low order numerical integration formulas for rigid and flexible multibody dynamics. In: Bottasso CL, Masarati P, Trainelli L (eds) Conference Proceedings of Multibody Dynamics 2007, ECCOMAS Thematic Conference, Milano, Italy, 25-28 June 2007

9. Negrut D, Rampalli R, Ottarsson G, Sajdak A (2007) On an implementation of the HHT method in the context of index 3 differential algebraic equations of multibody dynamics. ASME J. Comp. Nonlin. Dyn. 2: 73-85 
10. Shabana AA (2005) Dynamics of multibody systems. Cambridge University Press, third edition

11. Yen J, Petzold LR, Raha S (1998) A time integration algorithm for flexible mechanism dynamics: The DAE-alpha method. Comput. Methods Appl. Mech. Engrg. 158: 341-355 